\title{
Thermodynamics of dark energy interacting with dark matter and radiation
}

\author{
Mubasher Jamil, ${ }^{1, \text { f }}$ Emmanuel N. Saridakis, ${ }^{2, \text { 团 }}$ and M. R. Setare ${ }^{3, \text { 团 }}$ \\ ${ }^{1}$ Center for Advanced Mathematics and Physics, National University of Sciences and Technology, \\ Rawalpindi, 46000, Pakistan \\ ${ }^{2}$ Department of Physics, University of Athens, GR-157r71 Athens, Greece \\ ${ }^{3}$ Department of Science, Payame Noor University, Bijar, Iran
}

\begin{abstract}
We investigate the validity of the generalized second law of thermodynamics, in the cosmological scenario where dark energy interacts with both dark matter and radiation. Calculating separately the entropy variation for each fluid component and for the apparent horizon itself, we show that the generalized second law is always and generally valid, independently of the specific interaction form, of the fluids equation-of-state parameters and of the background geometry.

PACS numbers: 95.36.+x, 98.80.-k
\end{abstract}

\section{INTRODUCTION}

Recent cosmological observations obtained by SNe Ia [1], WMAP 2], SDSS [3] and X-ray [4] indicate that the observable universe experiences an accelerated expansion. Although the simplest way to explain this behavior is the consideration of a cosmological constant [5], the known fine-tuning problem [6] led to the dark energy paradigm. The dynamical nature of dark energy, at least in an effective level, can originate from a variable cosmological "constant" [7], or from various fields, such is a canonical scalar field (quintessence) [8], a phantom field, that is a scalar field with a negative sign of the kinetic term [9], or the combination of quintessence and phantom in a unified model named quintom [10]. Finally, an interesting attempt to probe the nature of dark energy according to some basic quantum gravitational principles is the holographic dark energy paradigm [11] (although the recent developments in Horava gravity could offer a dark energy candidate with perhaps better quantum gravitational foundations [12]).

The aforementioned models offer a satisfactory description of the dark-energy behavior and its observable features. However, attributing a dynamical nature to dark energy, these scenarios introduce a new cosmological problem [6], namely why are the densities of vacuum energy and dark matter nearly equal today since these scale very differently during the expansion history. The elaboration of this "coincidence" problem led to the consideration of generalized versions of the aforementioned models with the inclusion of a coupling between dark energy and dark matter. Thus, various forms of "interacting" dark energy models have been constructed in order to fulfil the observational requirements, including interacting quintessence [13], interacting phantom [14], interacting Chaplygin gas 15] and interacting holographic dark energy [16 18]. However, one could alleviate the coin-

\footnotetext{
*Electronic address: mjamil@camp.nust.edu.pk

${ }^{\dagger}$ Electronic address: msaridak@phys.uoa.gr

${ }^{\ddagger}$ Electronic address: rezakord@ipm.ir
}

cidence problem in the variable cosmological "constant" framework, too [19]. Finally, since the universe also comprises radiation, one can consider the scenario in which dark energy interacts with both dark matter and radiation [20]. We mention that the coupling to radiation is not new, since it is also in the center of "warm inflation" paradigm 21]. The motivation behind this extra interaction is the so-called 'triple coincidence problem' [22], that is it can offer an explanation to the fact that the radiation energy density is today only three orders of magnitude smaller than the dark-matter and dark-energy ones, although it also scales very differently. In particular, given the dark-energy-dark-matter coupling, we consider that the energy dissipated in this interaction is transferred to the radiation component and vice versa.

In the present work we are interested in investigating the interaction of dark energy with both dark matter and radiation fluids from the thermodynamic point of view. In particular, we desire to examine under what conditions the underlying system obeys the generalized second law of thermodynamics, namely the sum of entropies of the individual components, including that of the background, to be positive. The plan of the work is as follows: In section [II, we construct the scenario where dark energy interacts with dark matter and radiation fluids. In section III], considering the universe as a system bounded by the apparent horizon, we study the generalized second law of thermodynamics for our cosmological model. Finally, section IV is devoted to the summary of the obtained results.

\section{DARK ENERGY INTERACTING WITH DARK MATTER AND RADIATION}

Lets us construct a scenario where dark energy interacts with both dark-matter and radiation fluids. Throughout the work we consider a spatially homogeneous and isotropic universe described by the FriedmannRobertson-Walker metric

$$
d s^{2}=-d t^{2}+a^{2}(t)\left(\frac{d r^{2}}{1-k r^{2}}+r^{2} d \Omega^{2}\right),
$$


where $k$ is $0,1,-1$ for flat, closed and open geometry respectively. Thus, the first Friedmann equation writes

$$
H^{2}+\frac{k}{a^{2}}=\frac{1}{3}\left(\rho_{D E}+\rho_{M}+\rho_{\chi}\right),
$$

where $H$ is the Hubble parameter and $\rho_{D E}, \rho_{M}$ and $\rho_{x}$ denote the energy densities for dark energy, dark matter and radiation respectively. For simplicity, throughout this work we are using units where $8 \pi G=1$. We mention that the aforementioned framework holds independently of the specific dark energy description.

In the case where the various constituents of the universe are allowed to interact, the conservation equations for their energy densities write:

$$
\begin{aligned}
& \dot{\rho}_{D E}+3 H\left(1+w_{D E}\right) \rho_{D E}=-Q^{\prime} \\
& \dot{\rho}_{M}+3 H\left(1+w_{M}\right) \rho_{M}=Q \\
& \dot{\rho}_{\chi}+3 H\left(1+w_{\chi}\right) \rho_{\chi}=Q^{\prime}-Q
\end{aligned}
$$

where a dot denotes the derivative with respect to cosmic time. In these equations we have inserted the equation-of-state parameters of the various cosmological constituents, defined as $w_{i}=p_{i} / \rho_{i}$, where $p_{i}$ is the pressure of the corresponding constituent $i$.

In expressions (3), $Q$ and $Q^{\prime}$ describe the interaction terms, which can have an arbitrary form. In addition, we do not restrict the sign of these terms, that is $Q<0$ corresponds to energy transfer from dark-matter sector to the other two constituents, $Q^{\prime}>0$ corresponds to energy transfer form dark-energy sector to the other two fluids, and $Q^{\prime}<Q$ corresponds to energy loss for radiation. Obviously, in the case $Q=Q^{\prime}$, we obtain the usual model where dark energy interacts only with the darkmatter sector [13]. Additionally, note that this case may effectively appear as a self-conserved dark energy, with a non-trivial equation of state mimicking quintessence or phantom, as in the $\Lambda$ XCDM scenario [7, 19]. Lastly, concerning the form of interaction one can use many ansatzes 23], but we prefer to remain as general as possible.

Finally, it proves convenient to generalize 17] and construct the equivalent effective uncoupled model, described by:

$$
\begin{aligned}
& \dot{\rho}_{D E}+3 H\left(1+w_{D E}^{\mathrm{eff}}\right) \rho_{D E}=0 \\
& \dot{\rho}_{M}+3 H\left(1+w_{M}^{\mathrm{eff}}\right) \rho_{M}=0 \\
& \dot{\rho}_{\chi}+3 H\left(1+w_{\chi}^{\mathrm{eff}}\right) \rho_{\chi}=0
\end{aligned}
$$

where the effective equation-of-state parameters are given by

$$
\begin{aligned}
w_{D E}^{\mathrm{eff}} & =w_{D E}+\frac{Q^{\prime}}{3 H \rho_{D E}} \\
w_{M}^{\mathrm{eff}} & =w_{M}-\frac{Q}{3 H \rho_{M}} \\
w_{\chi}^{\mathrm{eff}} & =w_{\chi}+\frac{Q-Q^{\prime}}{3 H \rho_{\chi}} .
\end{aligned}
$$

\section{GENERALIZED SECOND LAW OF THERMODYNAMICS}

In the previous section we presented the cosmological scenario in which the dark-energy sector interacts with the dark-matter and radiation ones. In the present section we proceed to an investigation of its thermodynamic properties, and in particular of the generalized second thermodynamic law 24].

In the literature, when one desires to examine the thermodynamic behavior of a cosmological scenario, one considers the universe as a thermodynamical system. However, it is not trivial what 'volume' must be used, and in particular what 'radius', in order to acquire a consistent description. This subject becomes more important under the light of use of black-hole physics 25] in a cosmological framework [26], that is connecting the 'radius' and 'area' of the universe with its temperature and entropy respectively. For a flat geometry, using the Hubble horizon, which in this case coincides with the apparent horizon, as the aforementioned universe 'radius', one can extract the Friedmann equations by applying the first law of thermodynamics [27]. However, in order to achieve the same equivalence in a non-flat FRW geometry, one has to use the apparent horizon, since the use of Hubble horizon (in this case the two horizons do not coincide) cannot lead to a physical result 28]. The dynamical apparent horizon, a marginally trapped surface with vanishing expansion, is in general determined by the relation $h^{i j} \partial_{i} \tilde{r} \partial_{j} \tilde{r}=0$, which implies that the vector $\nabla \tilde{r}$ is null (or degenerate) on the apparent horizon surface [29]. In a metric of the form $d s^{2}=h_{i j} d x^{i} d x^{j}+\tilde{r}^{2} d \Omega_{2}^{2}$, with $h_{i j}=\operatorname{diag}\left(-1, a^{2} /\left(1-k r^{2}\right)\right), i, j=0,1$, it writes [29]:

$$
\tilde{r}_{A}=\frac{1}{\sqrt{H^{2}+\frac{k}{a^{2}}}} .
$$

Furthermore, for a dynamical spacetime the apparent horizon has been argued to be a causal horizon and it is associated with the gravitational entropy and surface gravity [29, 30]. Therefore, for the purpose of this work, we consider the universe as a thermodynamical system with the apparent horizon surface being its boundary.

The main goal of the present work is to examine the validity of the generalized second law of thermodynamics. As we have already mentioned, the Friedmann equations themselves arise straightaway from the first law of thermodynamics. Although this has been shown in the literature for only one fluid [28 31], it can be easily extended to the case at hand. Thus, one could equivalently construct the scenario of section $\Pi$ based solely on thermodynamics, with the only external input still needed, in order to close the equations system, being the information about the fluid content of the universe, and in particular the (three in our case) conservation equations 
(3).

Let us now proceed to the investigation of the generalized second law of thermodynamics in the universe. We are going to examine whether the sum of the entropy enclosed by the apparent horizon and the entropy of the apparent horizon itself, is not a decreasing function of time. Simple arguments suggest that after equilibrium establishes and the universe background geometry becomes FRW, all the fluids in the universe acquire the same temperature $T$ [18], which is moreover equal to the temperature of the horizon $T_{h}$ [27, 28], otherwise the energy flow would deform this geometry 32 .

In general, the apparent horizon $\tilde{r}_{A}$ is a function of time. Thus, a change $d \tilde{r}_{A}$ in time $d t$ will lead to a volume-change $d V$, while the energy and entropy will change by $d E$ and $d S$ respectively. However, since in the two states there is a common source $T_{\mu \nu}$, we can consider that the pressure $P$ and the temperature $T$ remain the same [27, 28]. Such a consideration is standard in thermodynamics, where one considers two equilibrium states differing infinitesimally in the extensive variables like entropy, energy and volume, while having the same values for the intensive variables like temperature and pressure. In this case the first law of thermodynamics writes $T d S=d E+P d V$, and therefore the dark-energy and dark-matter entropies read [33]:

$$
\begin{aligned}
d S_{D E} & =\frac{1}{T}\left(P_{D E} d V+d E_{D E}\right) \\
d S_{M} & =\frac{1}{T}\left(P_{M} d V+d E_{M}\right) \\
d S_{\chi} & =\frac{1}{T}\left(P_{\chi} d V+d E_{\chi}\right),
\end{aligned}
$$

where $V=4 \pi \tilde{r}_{A}^{3} / 3$ is the volume of the system bounded by the apparent horizon and thus $d V=4 \pi \tilde{r}_{A}^{2} d \tilde{r}_{A}$. In the aforementioned expressions we have also added the corresponding relation for radiation. We mention that due to equilibration, all the constituents (fluids) of the universe have the same temperature, while their energy and pressure are in general different. Finally, it proves useful to divide (7) by $d t$, obtaining:

$$
\begin{aligned}
\dot{S}_{D E} & =\frac{1}{T}\left(P_{D E} 4 \pi \tilde{r}_{A}^{2} \dot{\tilde{r}}_{A}+\dot{E}_{D E}\right) \\
\dot{S}_{M} & =\frac{1}{T}\left(P_{M} 4 \pi \tilde{r}_{A}^{2} \dot{\tilde{r}}_{A}+\dot{E}_{M}\right) \\
\dot{S}_{\chi} & =\frac{1}{T}\left(P_{\chi} 4 \pi \tilde{r}_{A}^{2} \dot{\tilde{r}}_{A}+\dot{E}_{\chi}\right),
\end{aligned}
$$

where

$\dot{\tilde{r}}_{A}=\frac{1}{2} H \tilde{r}_{A}^{3}\left[\left(1+w_{D E}\right) \rho_{D E}+\left(1+w_{M}\right) \rho_{M}+\left(1+w_{\chi}\right) \rho_{\chi}\right]$,

as it easily arises differentiating the Friedmann equation $\frac{1}{\tilde{r}_{A}^{2}}=\frac{1}{3}\left(\rho_{D E}+\rho_{M}+\rho_{\chi}\right)$ and using (3).
In order to connect the thermodynamically relevant quantities, namely the energies $E_{i}$ and pressures $P_{i}$, with the cosmologically relevant ones, namely the energy densities $\rho_{i}$ and the pressures $p_{i}$, we can straightforwardly use:

$$
\begin{aligned}
E_{D E} & =\frac{4 \pi}{3} \tilde{r}_{A}^{3} \rho_{D E} \\
E_{M} & =\frac{4 \pi}{3} \tilde{r}_{A}^{3} \rho_{M} \\
E_{\chi} & =\frac{4 \pi}{3} \tilde{r}_{A}^{3} \rho_{\chi},
\end{aligned}
$$

and

$$
\begin{aligned}
P_{D E} & =w_{D E}^{\mathrm{eff}} \rho_{D E} \\
P_{M} & =w_{M}^{\mathrm{eff}} \rho_{M} \\
P_{\chi} & =w_{\chi}^{\text {eff }} \rho_{\chi} .
\end{aligned}
$$

Note that expressions (11) arise necessarily from the "uncoupled" form of the system (relations (4) and (5)), since one needs an (effective or not) non-interacting system in order to apply basic thermodynamics and avoid concepts like the chemical potential. Inserting the time-derivatives of (10), along with (11), into (8), and using (3), we obtain:

$$
\begin{aligned}
\dot{S}_{D E} & =\frac{1}{T}\left(1+w_{D E}^{\mathrm{eff}}\right) \rho_{D E} 4 \pi \tilde{r}_{A}^{2}\left(\dot{\tilde{r}}_{A}-H \tilde{r}_{A}\right) \\
\dot{S}_{M} & =\frac{1}{T}\left(1+w_{M}^{\mathrm{eff}}\right) \rho_{M} 4 \pi \tilde{r}_{A}^{2}\left(\dot{\tilde{r}}_{A}-H \tilde{r}_{A}\right) \\
\dot{S}_{\chi} & =\frac{1}{T}\left(1+w_{\chi}^{\mathrm{eff}}\right) \rho_{\chi} 4 \pi \tilde{r}_{A}^{2}\left(\dot{\tilde{r}}_{A}-H \tilde{r}_{A}\right)
\end{aligned}
$$

At this stage, we have to connect the temperature of the fluids $T$, which is equal to that of the horizon $T_{h}$, with the geometry of the universe. According to the generalization of black hole thermodynamics [25] to a cosmological framework, the temperature of the horizon is related to its radius through [26, 28]

$$
T_{h}=\frac{1}{2 \pi \tilde{r}_{A}}
$$

Finally, concerning the entropy of the horizon, one can define it as [26, 28] $S_{h}=4 \pi \tilde{r}_{A}^{2} /(4 G)$, and since in this work we are using units where $8 \pi G=1$, we acquire $S_{h}=$ $8 \pi^{2} \tilde{r}_{A}^{2}$. Hence, we obtain:

$$
\dot{S}_{h}=16 \pi^{2} \tilde{r}_{A} \dot{\tilde{r}}_{A}
$$

Let us now proceed to the calculation of the total entropy variation. Adding relations (12)-(14) and (16), and substituting the effective equation-of-state parameters through (5), we find: 


$$
\dot{S}_{t o t} \equiv \dot{S}_{D E}+\dot{S}_{M}+\dot{S}_{\chi}+\dot{S}_{h}=8 \pi^{2} \tilde{r}_{A}^{3}\left(\dot{\tilde{r}}_{A}-H \tilde{r}_{A}\right)\left[\left(1+w_{D E}\right) \rho_{D E}+\left(1+w_{M}\right) \rho_{M}+\left(1+w_{\chi}\right) \rho_{\chi}\right]+16 \pi^{2} \tilde{r}_{A} \dot{\tilde{r}}_{A}
$$

Note that the aforementioned expression was simplified due to the useful relation

$$
\begin{array}{r}
\left(1+w_{D E}^{\mathrm{eff}}\right) \rho_{D E}+\left(1+w_{M}^{\mathrm{eff}}\right) \rho_{M}+\left(1+w_{\chi}^{\mathrm{eff}}\right) \rho_{\chi}= \\
\left(1+w_{D E}\right) \rho_{D E}+\left(1+w_{M}\right) \rho_{M}+\left(1+w_{\chi}\right) \rho_{\chi} .
\end{array}
$$

Thus, substituting also $\dot{\tilde{r}}_{A}$ by (9) we result to:

$$
\dot{S}_{t o t}=4 \pi^{2} \tilde{r}_{A}^{6} H\left[\left(1+w_{D E}\right) \rho_{D E}+\left(1+w_{M}\right) \rho_{M}+\left(1+w_{\chi}\right) \rho_{\chi}\right]^{2} \geq 0 .
$$

(19) provides the expression of the generalized second law of thermodynamics in the scenario where dark energy interacts with dark matter and radiation. The fact that $\dot{S}_{\text {tot }}$ is always non-negative proves the validity of this law at all cosmological times. We mention that this result holds independently of the interaction form, of the fluids equation-of-state parameters, and of the background geometry, provided it is FRW. Finally, note that in the aforementioned analysis we have not taken into account the possible black-hole formation in the universe and its effect on entropy.

Let us make a comment here for completeness. As we observe from (19), there is a critical value of the darkenergy equation-of-state parameter that leads to $\dot{S}_{t o t}=$ 0 . In particular this happens if $w_{D E c r}=-1-(1+$ $\left.w_{M}\right) \rho_{M} / \rho_{D E}-\left(1+w_{\chi}\right) \rho_{\chi} / \rho_{D E}$. That is, for regular dark-matter and radiation, $w_{D E c r}$ lies in the phantom regime. However, in order for $w_{D E}$ to obtain this value one definitely needs a short of fine tuning. Its calculation at present (where the corresponding density parameters are $\Omega_{M} \approx 0.72, \Omega_{M} \approx 0.28, w_{M} \approx 0$ and neglecting radiation for simplicity) leads to $w_{D E c r} \approx-1.39$, which is outside the observational intervals for $w_{D E}$. But this could change in the future, if $\Omega_{M}$ decrease sufficiently. We mention that if $w_{D E}$ is even smaller, then $\dot{S}_{t o t}$ will become positive again. Finally, from (9) we observe that this value for $w_{D E}=w_{D E c r}$ leads also to $\dot{\tilde{r}}_{A}=0$. Thus, from the apparent horizon definition (6) we can easily calculate the corresponding solution for the scale factor, namely $a(t)=e^{\alpha t+\beta}+4 k e^{-\alpha t-\beta}$ and $a(t)=e^{-\alpha t-\beta}+$ $4 k e^{\alpha t+\beta}$, where $\alpha, \beta$ are constants, and with the second solution existing only for a closed universe. Obviously, for large times these solution tend to a de Sitter solution. Note however that if there is a strong energy-transfer from dark energy to the other sectors, the former will not necessarily dominate the universe completely.

In the following we discuss about the sign of the temperature and entropy. In general, if the total equation- of-state parameter of the universe lies above the phantom divide, then both these quantities are unambiguously positive as usual. However, if the universe lies in the phantom phase the subject is still open in the literature. Assuming a zero chemical potential the temperature must be negative, with the density and the entropy positive [34] and assuming a negative chemical potential then temperature, entropy and density are positive [35]. Additionally, in 36] it was shown that even with an arbitrary chemical potential the temperature of a phantom universe is negative, with the density and the entropy positive, while in [32] it was found that the phantom temperature is positive and its entropy negative. Finally, in 37] it was argued that one can describe the phantom universe either with negative temperature and positive entropy, or with negative entropy and positive temperature. In the analysis of this work equilibrium requires all the fluids in the universe to have the same temperature, which is moreover equal to the temperature of the horizon (although one could put into question the equilibrium assumption and examine the non-equilibrium case too). Since the horizon temperature is always positive, it is deduced that the universe temperature will be positive even if it lies in the phantom phase. Thus, in order to be in agreement with the literature, one should have a negative universe entropy in this case. Note however that the negative entropy of the universe ingredients is overcome by the positive horizon entropy, and thus the total entropy is always positive.

In particular, and assuming complete dark energy domination for simplicity, in the quintessence regime we have $\dot{\tilde{r}}_{A}>0$ (according to (9)). Thus, according to (16) we obtain $\dot{S}_{h}>0$, while according to (12) we have $\dot{S}_{D E}<0$. Furthermore, $S_{h}>0$ (as always), while $S_{D E}>0$ (since we are in the quintessence regime). Thus, in this regime we have $\dot{S}_{t o t}>0$ (according to (19)) and $S_{t o t}>0$. On the other hand, in the phantom regime (9) leads to $\dot{\tilde{r}}_{A}<0$, and thus (16) gives $\dot{S}_{h}<0$, while 
(12) gives $\dot{S}_{D E}>0$. Moreover, $S_{h}>0$ (as always), while $S_{D E}<0$ (since we are in the phantom regime). However, in this case we obtain $S_{t o t}>0$ and also $\dot{S}_{t o t}>0$. So in summary, the present work and the validity of the generalized second law, is consistent with the positivetemperature and negative-entropy picture of the phantom dark energy. Finally, note that the inconsistency of the generalized second law with the negative-temperature and positive-entropy picture of the phantom dark energy, was already mentioned in 38].

Before closing this section let us make a comment on the horizon we use in the present work. Concerning the first law of thermodynamics it has been proven in 39] that the field equations of any (diffeomorphism invariant) gravitational theory must be expressible as a thermodynamic identity, $T d S=d E$, around any event in the spacetime. However, concerning the generalized second law of thermodynamics, it has been shown that it is generally valid only if one uses the apparent horizon [28 31], while it is conditionally valid for other horizon choices [38]. Thus, repeating our calculations using the future event horizon $R_{h}=\int_{a}^{\infty} d a /\left(H a^{2}\right)$ instead of the apparent horizon $r_{A}$, we find that the generalized second law is not always valid. Clearly, the separate investigation of each horizon is a crucial and open subject in the thermodynamic aspects of gravity, as it is discussed in detail in the recent review [40].

\section{CONCLUSIONS}

In this work we investigated the cosmological scenario where dark energy interacts with both dark matter and radiation, a scenario which could alleviate the triple coincidence problem 22]. After reminding that in such fluid cosmological models the Friedmann equations can arise from the first law of thermodynamics, with the fluid conservation equations being the only external input re- quired to close the equations system, we examined the validity of the generalized second law of thermodynamics. Considering the universe as a thermodynamical system bounded by the apparent horizon, and calculating separately the entropy variation for each fluid component and for the horizon itself, we resulted to an expression for the time derivative of the total entropy of the universe.

According to our main result, that is expression (19), the time derivative of the total entropy is always nonnegative, and this holds independently of the specific interaction form, of the fluids equation-of-state parameters, and of the background geometry. Thus, the generalized second law of thermodynamics is always and generally valid, as long as one considers the apparent horizon as the universe "radius" (the use of other choices, such is the future event horizon, leads to conditional validity only). However, we mention that the present work is consistent with the positive-temperature and negative-entropy picture of the phantom dark energy [37]. Finally, it is interesting to notice that if the dark-energy equation-ofstate parameter takes a critical phantom value, then the total entropy of the universe remains constant.

In the present work we have proven the validity of the generalized second law of thermodynamic in the scenario where dark energy interacts with dark-matter and radiation sectors. This result is necessary for the consideration of such scenarios, but it is not sufficient. One must also examine whether such models affect the known cosmological epochs, before proceeding to their safe use. But such a study is beyond the purpose of this work and it is left for future investigation.

\section{Acknowledgments}

The authors would like to thank R. Horvat and H. Mohseni Sadjadi for useful discussions, and an anonymous referee for fruitful comments and advices.
[1] A. G. Riess et al. [Supernova Search Team Collaboration], Astron. J. 116, 1009 (1998); S. Perlmutter et al. [Supernova Cosmology Project Collaboration], Astrophys. J. 517, 565 (1999).

[2] C. L. Bennett et al., Astrophys. J. Suppl. 148, 1 (2003).

[3] M. Tegmark et al. [SDSS Collaboration], Phys. Rev. D 69, 103501 (2004).

[4] S. W. Allen, et al., Mon. Not. Roy. Astron. Soc. 353, 457 (2004).

[5] V. Sahni and A. Starobinsky, Int. J. Mod. Phy. D 9, 373 (2000); P. J. Peebles and B. Ratra, Rev. Mod. Phys. 75, 559 (2003).

[6] P. J. Steinhardt, Critical Problems in Physics (1997), Princeton University Press.

[7] J. Sola and H. Stefancic, Phys. Lett. B 624, 147 (2005); J. Sola and H. Stefancic, Mod. Phys. Lett. A 21, 479 (2006); I. L. Shapiro and J. Sola, Phys. Lett. B 682, 105
(2009).

[8] B. Ratra and P. J. E. Peebles, Phys. Rev. D 37, 3406 (1988); C. Wetterich, Nucl. Phys. B 302, 668 (1988); A. R. Liddle and R. J. Scherrer, Phys. Rev. D 59, 023509 (1999); I. Zlatev, L. M. Wang and P. J. Steinhardt, Phys. Rev. Lett. 82, 896 (1999); Z. K. Guo, N. Ohta and Y. Z. Zhang, Mod. Phys. Lett. A 22, 883 (2007); S. Dutta, E. N. Saridakis and R. J. Scherrer, Phys. Rev. D 79, 103005 (2009).

[9] R. R. Caldwell, Phys. Lett. B 545, 23 (2002); R. R. Caldwell, M. Kamionkowski and N. N. Weinberg, Phys. Rev. Lett. 91, 071301 (2003); S. Nojiri and S. D. Odintsov, Phys. Lett. B 562, 147 (2003); V. K. Onemli and R. P. Woodard, Phys. Rev. D 70, 107301 (2004); M. R. Setare, J. Sadeghi, A. R. Amani, Phys. Lett. B 666, 288, (2008); M. R. Setare and E. N. Saridakis, JCAP 0903, 002 (2009); E. N. Saridakis, Nucl. Phys. B 819, 
116 (2009).

[10] B. Feng, X. L. Wang and X. M. Zhang, Phys. Lett. B 607, 35 (2005); Z. K. Guo, et al., Phys. Lett. B 608, 177 (2005); M.-Z Li, B. Feng, X.-M Zhang, JCAP, 0512, 002 (2005); B. Feng, M. Li, Y.-S. Piao and X. Zhang, Phys. Lett. B 634, 101 (2006); M. R. Setare, Phys. Lett. B 641, 130 (2006); W. Zhao and Y. Zhang, Phys. Rev. D 73, 123509 (2006); M. R. Setare, J. Sadeghi, and A. R. Amani, Phys. Lett. B 660, 299 (2008); M. R. Setare and E. N. Saridakis, Phys. Lett. B 668, 177 (2008); M. R. Setare and E. N. Saridakis, JCAP 0809, 026 (2008); M. R. Setare and E. N. Saridakis, Int. J. Mod. Phys. D 18, 549 (2009).

[11] S. D. H. Hsu, Phys. Lett. B 594, 13 (2004); M. Li, Phys. Lett. B 603, 1 (2004); Q. G. Huang and M. Li, JCAP 0408, 013 (2004); M. Ito, Europhys. Lett. 71, 712 (2005); X. Zhang and F. Q. Wu, Phys. Rev. D 72, 043524 (2005); D. Pavon and W. Zimdahl, Phys. Lett. B 628, 206 (2005); S. Nojiri and S. D. Odintsov, Gen. Rel. Grav. 38, 1285 (2006); E. Elizalde, S. Nojiri, S. D. Odintsov and P. Wang, Phys. Rev. D 71, 103504 (2005); H. Li, Z. K. Guo and Y. Z. Zhang, Int. J. Mod. Phys. D 15, 869 (2006); E. N. Saridakis, Phys. Lett. B 660, 138 (2008); E. N. Saridakis, JCAP 0804, 020 (2008); E. N. Saridakis, Phys. Lett. B 661, 335 (2008).

[12] P. Horava, Phys. Rev. D 79, 084008 (2009); G. Calcagni, arXiv:0904.0829 [hep-th]; E. Kiritsis and G. Kofinas, Nucl. Phys. B 821, 467 (2009); H. Lu, J. Mei and C. N. Pope, arXiv:0904.1595 [hep-th]; C. Charmousis, G. Niz, A. Padilla and P. M. Saffin, arXiv:0905.2579 [hep-th]; E. N. Saridakis, arXiv:0905.3532 [hep-th]; X. Gao, Y. Wang, R. Brandenberger and A. Riotto, arXiv:0905.3821 [hep-th]; M. i. Park, arXiv:0905.4480 [hep-th]; Y. F. Cai and E. N. Saridakis, arXiv:0906.1789 [hep-th]; M. Botta-Cantcheff, N. Grandi and M. Sturla, arXiv:0906.0582 [hep-th]; M. R. Setare, arXiv:0909.0456 [hep-th]; C. Germani, A. Kehagias and K. Sfetsos, JHEP 0909, 060 (2009); G. Leon and E. N. Saridakis, JCAP 0911, 006 (2009).

[13] C. Wetterich, Astron. Astrophys. 301, 321 (1995) L. Amendola, Phys. Rev. D 60, 043501 (1999); A. P. Billyard and A. A. Coley, Phys. Rev. D 61, 083503 (2000); A. Nunes, J. P. Mimoso and T. C. Charters, Phys. Rev. D 63, 083506 (2001); G. R. Farrar and P. J. E. Peebles, Astrophys. J. 604, 1 (2004); D. F. Mota and C. van de Bruck, Astron. Astrophys. 421, 71 (2004); X. Zhang, Mod. Phys. Lett. A 20, 2575 (2005); T. Gonzalez, G. Leon and I. Quiros, Class. Quant. Grav. 23, 3165 (2006); T. Clifton and J. D. Barrow, Phys. Rev. D 73, 104022 (2006); M. Manera and D. F. Mota, Mon. Not. Roy. Astron. Soc. 371, 1373 (2006); T. Clifton and J. D. Barrow, Phys. Rev. D 75, 043515 (2007).

[14] Z. K. Guo, R. G. Cai and Y. Z. Zhang, JCAP 0505, 002 (2005); Z. K. Guo and Y. Z. Zhang, Phys. Rev. D 71, 023501 (2005); R. Curbelo, T. Gonzalez, G. Leon and I. Quiros, Class. Quant. Grav. 23, 1585 (2006); J. D. Barrow and T. Clifton, Phys. Rev. D 73, 103520 (2006) T. Gonzalez and I. Quiros, Class. Quant. Grav. 25, 175019 (2008); X. m. Chen, Y. g. Gong and E. N. Saridakis, JCAP 0904, 001 (2009).

[15] H. Garcia-Compean, G. Garcia-Jimenez, O. Obregon and C. Ramirez, JCAP 0807, 016 (2008); M. Jamil and M. A. Rashid, Eur. Phys. J. C 60, 141 (2009).

[16] B. Wang, Y. G. Gong and E. Abdalla, Phys. Lett. B 624,
141 (2005); M. R. Setare, Phys. Lett. B 642, 1 (2006); B. Hu and Y. Ling, Phys. Rev. D 73, 123510 (2006).

[17] H. Kim, H. W. Lee and Y. S. Myung, Phys. Lett. B 632, 605 (2006).

[18] M. R. Setare, JCAP 0701, 023 (2007).

[19] J. Grande, J. Sola and H. Stefancic, JCAP 0608, 011 (2006); J. Grande, A. Pelinson and J. Sola, Phys. Rev. D 79, 043006 (2009).

[20] N. Cruz, S. Lepe and F. Pena, Phys. Lett. B 663, 338 (2008).

[21] A. Berera, Phys. Rev. Lett. 75, 3218 (1995); A. Berera, M. Gleiser and R. O. Ramos, Phys. Rev. Lett. 83, 264 (1999); J. M. F. Maia and J. A. S. Lima, Phys. Rev. D 60, 101301 (1999); J. P. Mimoso, A. Nunes and D. Pavon, Phys. Rev. D 73, 023502 (2006).

[22] N. Arkani-Hamed, L. J. Hall, C. F. Kolda and H. Murayama, Phys. Rev. Lett. 85, 4434 (2000).

[23] L. P. Chimento, A. S. Jakubi, D. Pavon and W. Zimdahl, Phys. Rev. D 67, 083513 (2003); C. G. Böhmer, G. Caldera-Cabral, R. Lazkoz and R. Maartens, Phys. Rev. D 78, 023505 (2008).

[24] W. G. Unruh and R. M. Wald, Phys. Rev. D 25, 942 (1982); P. C. W. Davies, Class. Quant. Grav. 4, L225 (1987); H. Mohseni Sadjadi, Phys. Rev. D 76, 104024 (2007); R. Horvat, Phys. Lett. B 648, 374 (2007); M. R. Setare and E. C. Vagenas, Phys. Lett. B 666, 111 (2008); A. Sheykhi and B. Wang, Phys. Lett. B 678, 434 (2009).

[25] G. W. Gibbons and S. W. Hawking, Phys. Rev. D 15, 2738 (1977).

[26] T. Jacobson, Phys. Rev. Lett. 75, 1260 (1995); T. Padmanabhan, Phys. Rept. 406, 49 (2005); A. Paranjape, S. Sarkar and T. Padmanabhan, Phys. Rev. D 74, 104015 (2006).

[27] A. V. Frolov and L. Kofman, JCAP 0305, 009 (2003); U. H. Danielsson, Phys. Rev. D 71, 023516 (2005); R. Bousso, Phys. Rev. D 71, 064024 (2005).

[28] R. G. Cai and S. P. Kim, JHEP 0502, 050 (2005); M. Akbar and R. G. Cai, Phys. Rev. D 75, 084003 (2007).

[29] D. Bak and S. J. Rey, Class. Quant. Grav. 17, L83 (2000).

[30] S. A. Hayward, Class. Quant. Grav. 15, 3147 (1998); S. A. Hayward, S. Mukohyama and M. C. Ashworth, Phys. Lett. A 256, 347 (1999).

[31] A. Sheykhi, B. Wang and R. G. Cai, Nucl. Phys. B 779, 1 (2007).

[32] G. Izquierdo and D. Pavon, Phys. Lett. B 633, 420 (2006).

[33] B. Wang, Y. Gong and E. Abdalla, Phys. Rev. D 74, 083520 (2006); Y. Gong, B. Wang and A. Wang, Phys. Rev. D 75, 123516 (2007).

[34] P. F. Gonzalez-Diaz and C. L. Siguenza, Nucl. Phys. B 697, 363 (2004); Y. S. Myung, Phys. Lett. B 671, 216 (2009).

[35] J. A. S. Lima and S. H. Pereira, Phys. Rev. D 78, 083504 (2008); S. H. Pereira and J. A. S. Lima, Phys. Lett. B 669, 266 (2008).

[36] E. N. Saridakis, P. F. Gonzalez-Diaz and C. L. Siguenza, Class. Quant. Grav. 26, 165003 (2009).

[37] I. H. Brevik, S. Nojiri, S. D. Odintsov and L. Vanzo, Phys. Rev. D 70, 043520 (2004); S. Nojiri and S. D. Odintsov, Phys. Rev. D 70, 103522 (2004).

[38] H. Mohseni Sadjadi, Phys. Rev. D 73, 063525 (2006); R. Horvat, Phys. Lett. B 664, 201 (2008).

[39] T. Padmanabhan, arXiv:0903.1254 [hep-th]. 
[40] T. Padmanabhan, arXiv:0911.5004 [gr-qc]. 